\title{
SEX, AFFECTIVE TEMPERAMENTS AND INFORMATION STRESS
}

\section{WŁODZIMIERZ ONISZCZENKO and MARIA LEDZIŃSKA}

University of Warsaw, Warsaw, Poland

Faculty of Psychology, Department of Individual Differences

\begin{abstract}
Objectives: The main purpose of the study was to investigate the association between sex and the level of information stress, as mediated by affective temperaments. Material and Methods: The sample consisted of 231 healthy Caucasian adults (150 women and 81 men) recruited from a general population. The participants' age ranged $18-56$ years $(\mathrm{M} \pm \mathrm{SD}=25.07 \pm 6.36)$. Affective temperaments were assessed using the Temperament Evaluation of Memphis, Pisa, Paris and San Diego Auto-questionnaire (TEMPS-A). To assess the level of information stress, the Information Stress Questionnaire (ISQ) was used. Results: Information stress displayed low to medium positive correlations with depressive, cyclothymic, irritable and anxious temperaments, and a negative correlation with the hyperthymic temperament. The female group was characterized with significantly higher age, information stress, and anxious temperament values, and with a significantly lower irritable temperament value, when compared to males. Cyclothymic temperament, anxious temperament and hyperthymic temperament were found to be significant predictors of information stress. The mediation analysis showed a significant direct effect of sex on information stress. The anxious temperament was a significant mediator of the relationship between sex and information stress. Conclusions: The results showed the relationship between sex and information stress, including the role of anxious temperament as a mediator. Int J Occup Med Environ Health. 2019;32(5):635-44
\end{abstract}

Key words:

adults, affective temperament, sex, sex difference, information stress, mediation analysis

\section{INTRODUCTION}

The contemporary world is characterized by the global flow of a great deal of information. This phenomenon is also referred to as information overload or cognitive overload syndrome, which is related not only to the amount of information and the available processing time, but also to the characteristics of information, such as novelty, complexity and uncertainty [1].

Information stress (abbreviated as info stress) is a form of psychological stress in which the stressor is an excess of information, such as information overload [2]. It occurs when an individual experiences a surge of information, i.e., when the amount of signals in the environment exceeds the capabilities of reception and processing [3]. When individuals are exposed to more information than they can effectively use, the information overload may cause stress, frustration and dissatisfaction [4]. It is worth emphasizing that many researchers point to stress as a consequence of information overload for which one's coping strategies are ineffective [5-7]. Information overload can also cause information anxiety resulting from the inability to access, understand, or make use of necessary information $[1,8,9]$. Haase et al. [10] suggested that the capacity for tolerating information overload might be partially related to the strength of excitation and mobility as temperamental traits based on Pavlov's typology of central nervous system

Funding: this research was supported by the University of Warsaw (grant No. BST 1868-2018, entitled "Temperament in terms of the Regulative Theory of Temperament, the concept of affective temperaments and Dark Triad, and procrastination," grant manager: Prof. Włodzimierz Oniszczenko).

Received: October 9, 2018. Accepted: May 13, 2019.

Corresponding author: Włodzimierz Oniszczenko, University of Warsaw, Faculty of Psychology, Department of Individual Differences, Stawki 5-7, 00-183 Warsaw, Poland (e-mail:wlodek@psych.uw.edu.pl) 
properties. Furthermore, Ledzińska and Postek [11] suggested a moderate correlation between information stress experience and a lower stimulation processing capacity which is temperamentally determined.

Important factors regulating human reactions to stress, such as depression and anxiety, may serve affective temperaments. Affective temperament types (depressive, cyclothymic, hyperthymic, irritable, and anxious) are trait-related manifestations that play a fundamental role in the predisposition to affective disorders and affective psychoses [12,13]. Kawamura et al. [14] showed that affective temperaments exhibit good long-term stability and might be considered stable traits. It is important that affective temperaments encompass healthy personality traits [15] and have both universal and culturally specific characteristics, as Vázquez et al. [16] proposed. Studies confirm the similarity between the concept of affective temperaments and other models of personality, including normally functioning personality (temperament). For example, Blöink et al. [17] showed positive correlations between neuroticism, as measured by the NEO-Five Factor Inventory, and all the affective temperaments except hyperthymic. In turn, Kwapil et al. [18] showed that 5 factor personality model traits accounted for $38 \%$ (irritable temperament) to $49 \%$ (hyperthymic temperament) of the total variance in affective temperaments. Also, Oniszczenko et al. [19] demonstrated that 2 temperamental traits, emotional reactivity and perseveration, positively correlated with anxious, cyclothymic, depressive and irritable temperaments, predicting $2 \%$ (irritable temperament) to $24 \%$ (anxious temperaments) of the variance in affective temperaments.

Emotional reactivity and perseveration are the traits conducive to intense emotional response and prolonged emotional arousal, respectively [20]. All these personality factors are believed to be associated with different aspects of the stress response. For example, people with higher levels of neuroticism are more likely to feel anxious, and expe- rience stress and negative affect [21]. Furthermore, this suggests that affective temperaments positively correlated with neuroticism, emotional reactivity and perseveration may also impact on how people perceive and experience stress.

As Rihmer et al. [22] have found, depressive, cyclothymic and anxious temperaments are more frequent in women, whereas hyperthymic and irritable temperaments predominate among men. Other authors have confirmed these results [17,19].

Experiences, skills and age have been indicated among the personal factors that contribute to information overload and its consequences, unlike sex or personality traits [1]. Nevertheless, some premises can predict the role of sex and affective temperaments in information stress. For example, Lungu et al. [23] showed that women were more sensitive to negative emotional images than men. Allan et al. [24] demonstrated higher levels of self-reported anxiety sensitivity in women than in men, while Bottesi et al. [25] showed higher levels of worry and intolerance of uncertainty in women, compared to men. In turn, Bale and Epperson [26] suggested that women might show an increased risk of affective disorders, including depression and anxiety, in adolescence and throughout adulthood.

The information stress resulting from the overproduction of data is mediated, as are other kinds of stress, by temperamental features. Four areas of temperamental ties to stress have been identified so far, i.e., the way the stressors are registered, the emotional and physiological experience of stress, the type and intensity of coping procedures employed, and, finally, the costs of a stressful situation (e.g., for health) [11,20]. Affective temperaments are genetically conditioned styles of emotional reaction, which may be the predisposing factor in the occurrence of information stress, and may play a significant role as a mediator between sex and information stress. As noted, women exhibit a higher level of depressive, cyclothymic, irritable and anxious temperaments as individual predictors of the 
intensity of stress experience, compared to men, so they may be more likely to experience information stress.

The main purpose of the study was to investigate the association between sex and the level of information stress, as mediated by affective temperaments. Taking into account the fact that women are more sensitive to anxiety stimuli and show a higher level of affective temperament, compared to men $[17,19,22]$, the authors hypothesized that sex might affect the level of information stress directly and indirectly, through the mediation of the affective temperaments. The authors expected that anxious temperament would play a crucial role in the mediation between sex and the level of information stress, and they assumed that individuals with high rates of anxious temperament would experience more stress and react strongly emotionally to stressors due to their uncontrollable worrying, hypervigilance, tension, and irritability [27].

The authors expect their study to expand knowledge about the importance of affective temperaments in response to information stress in both women and men, in the nonclinical population. To the best of their knowledge, this is the first attempt to relate affective temperaments and stress info, taking into account differences in affective temperaments between women and men.

\section{MATERIAL AND METHODS}

\section{Participants}

The sample consisted of 231 healthy Caucasian adults (150 women and $81 \mathrm{men}$ ) recruited from a general population. The participants' ages ranged 18-56 years $(\mathrm{M} \pm \mathrm{SD}=25.07 \pm 6.36)$. In terms of the educational level, 132 participants had post-secondary education, 94 participants had secondary education, and 2 participants had primary education ( 3 participants did not provide any information about their education). The study was part of a larger research project on personality. The participants were recruited directly by the psychologists, who contacted students of various faculties, their colleagues, friends, par- ents and, in the case of working students, their co-workers. The inclusion criteria were age $\geq 18$ years and an absence of self-reported mental problems.

The study was anonymous, and participation was voluntary. All the self-report questionnaires were administered in a standard manner. Informed consent was obtained from all the participants before they were included in the study, and they did not receive any compensation. The research project was approved by the local Research Ethics Commission at the Faculty of Psychology, University of Warsaw.

\section{Measurements}

Affective temperaments were assessed using the Polish version of the Temperament Evaluation of Memphis, Pisa, Paris, and San Diego Auto-Questionnaire (TEMPS-A) [28,29]. It was a self-reported instrument comprising 110 items (109 for men) with a yes/no response format. It was composed of 5 scales (the values of Cronbach's $\alpha$ for the Polish version are given in parentheses): depressive $(0.70)$, cyclothymic (0.77), hyperthymic (0.75), irritable (0.76) and anxious (0.83). For each answer, a "yes" response was scored as 1 , and a "no" response as 0 . These scores were added and divided by the number of items belonging to each affective temperament scale (21 for each of the first 4 categories of temperaments and 26 for the last category, i.e., anxious temperament).

To assess the level of information stress, the Information Stress Questionnaire (ISQ) developed by Ledzińska [30] was used. The ISQ is a diagnostic instrument of a discomfort experienced by an individual as a result of a metacognitive observation of not being able to deal with information overload at every stage of information processing. The ISQ was a self-report measure consisting of 45 items directly related to information stress, with a 4-point Likert scale (1-4). Higher scores indicated greater information stress intensity. Two examples of the items are "The excess of information in my environment causes me to have 
problems with focusing attention" and "Sometimes, I feel so overloaded by information that I am unable to remind myself of the important events of the day." The ISQ had a Cronbach's $\alpha$ value of 0.96 .

\section{Statistical analysis}

Statistical analysis was performed using IBM SPSS Statistics 24 [31]. Data normality was checked based on the skewness and kurtosis values, following the application of the criteria specified by Tabachnick and Fidell [32]. Since the compared groups were not equinumerous, the Mann-Whitney $U$ test for independent samples was used for comparing the male and female groups. The relationships between the variables were examined with Pearson product-moment coefficients. A hierarchical multiple regression analysis was used to estimate the effect of sex and affective temperaments as predictors of info stress ratings. The mediation analyses were conducted following Baron and Kenny's [33] approach, using the Sobel test for mediation.

\section{RESULTS}

The skewness and kurtosis analysis showed that all the variables were distributed normally, except age. Skewness varied from -0.14 (hyperthymic temperament) to 0.93 (irritable temperament), while kurtosis ranged from -0.60 (hyperthymic temperament) to 0.38 (irritable temperament). Skewness for age was 2.55, and kurtosis was 7.50.

Table 1 provides the descriptive statistics of the male and female groups, and the differences in the studied variables between the groups. The female group was characterized with significantly higher age, information stress and anxious temperament values, and with a significantly lower irritable temperament value, compared to the male group. However, no significant differences between these groups were noted in the depressive, cyclothymic, and hyperthymic temperaments.

Table 2 presents the Pearson's $r$ correlation coefficients between the age, information stress, and affective temperament traits. The results show that the correlations between all the variables studied were low to high (an ab-

Table 1. Descriptive statistics and gender differences for age, information stress and the Temperament Evaluation of Memphis, Pisa, Paris and San Diego Auto-Questionnaire Scales [29] in the whole sample ( $\mathrm{N}=231)$ in the study conducted in 2017-2018 in Warsaw

\begin{tabular}{|c|c|c|c|c|}
\hline \multirow[t]{2}{*}{ Variable } & \multicolumn{2}{|c|}{$\begin{array}{c}\text { Participants } \\
(\mathrm{N}=231) \\
(\mathrm{M} \pm \mathrm{SD})\end{array}$} & \multirow[t]{2}{*}{$\mathrm{Z}$} & \multirow[t]{2}{*}{ Cohen's d } \\
\hline & $\begin{array}{l}\text { females } \\
(\mathrm{N}=150)\end{array}$ & $\begin{array}{c}\text { males } \\
(\mathrm{N}=81)\end{array}$ & & \\
\hline Age & $25.46 \pm 6.34$ & $24.36 \pm 6.42$ & $-2.93^{* *}$ & 0.17 \\
\hline Information stress & $100.49 \pm 14.30$ & $94.85 \pm 17.72$ & $-2.40^{*}$ & 0.35 \\
\hline \multicolumn{5}{|c|}{$\begin{array}{l}\text { Temperament Evaluation of Memphis, Pisa, Paris } \\
\text { and San Diego Auto-Questionnaire scale }\end{array}$} \\
\hline depressive & $0.40 \pm 0.17$ & $0.35 \pm 0.14$ & -1.85 & 0.32 \\
\hline cyclothymic & $0.39 \pm 0.19$ & $0.37 \pm 0.20$ & -0.55 & 0.05 \\
\hline hyperthymic & $0.48 \pm 0.20$ & $0.49 \pm 0.20$ & -0.37 & -0.05 \\
\hline irritable & $0.21 \pm 0.17$ & $0.27 \pm 0.20$ & $-2.57^{* *}$ & -0.32 \\
\hline anxious & $0.37 \pm 0.22$ & $0.27 \pm 0.18$ & $-3.58^{* * *}$ & 0.50 \\
\hline
\end{tabular}

Z-value for the Mann-Whitney U test.

$* \mathrm{p}<0.05 ; * * \mathrm{p}<0.01 ; * * * \mathrm{p}<0.001$. 
Table 2. Pearson's r correlations between age, information stress and Temperament Evaluation of Memphis, Pisa, Paris and San Diego Auto-Questionnaire scales [29] in the whole sample $(\mathrm{N}=231)$ in the study conducted in 2017-2018 in Warsaw

\begin{tabular}{lcccccc}
\hline \multirow{2}{*}{ Variable } & \multicolumn{7}{c}{ Pearson's r correlations } \\
\cline { 2 - 7 } & 2 & 3 & 4 & 5 & 6 & 7 \\
\hline 1. Age & -0.07 & -0.08 & $-0.26^{* * *}$ & 0.06 & $-0.13^{*}$ & -0.08 \\
2. Information stress & & $0.29^{* * *}$ & $0.34^{* * *}$ & $-0.28^{* * *}$ & $0.13^{*}$ & $0.36^{* * *}$ \\
3. Depressive & & & $0.53^{* * *}$ & $-0.58^{* * *}$ & $0.34^{* * *}$ & $0.68^{* * *}$ \\
4. Cyclothymic & & & $-0.25^{* * *}$ & $0.59^{* * *}$ & $0.52^{* * *}$ \\
5. Hyperthymic & & & & -0.11 & $-0.35^{* * *}$ \\
6. Irritable & & & & & $0.42^{* * *}$ \\
7. Anxious & & & & & 1 \\
\hline
\end{tabular}

$* \mathrm{p}<0.05 ; * * * \mathrm{p}<0.001$.

solute value of $\mathrm{r}$ of 0.1 was classified as low, 0.3 as medium, and 0.5 as high) [34].

As Table 2 shows, information stress displayed low to medium positive correlations with depressive, cyclothymic, irritable and anxious temperaments, and a negative correlation with hyperthymic temperament. Medium to high positive correlations between depressive, cyclothymic, irritable and anxious temperaments were demonstrated. Low to high negative correlations between hyperthymic temperament and other affective temperaments were also observed. The participants' age displayed low negative correlations with cyclothymic and irritable temperaments.

No correlations between age and the other variables were observed.

To determine the extent to which sex and affective temperaments could be viewed as predictors of information stress, a hierarchical multiple regression analysis was conducted. Sex was entered in Step 1 of the hierarchical multiple regression analysis, explaining $2 \%$ of the information stress variance in the whole sample. When 5 affective temperaments were added in Step 2, the percentage of variance accounted for increased to $19 \%, \mathrm{~F}(5,224)=10.33$, $\mathrm{p}<0.001$. Based on the regression coefficients, cyclothymic temperament $(\beta=0.28, \mathrm{p}<0.001)$, anxious temperament $(\beta=0.25, p<0.01)$ and hyperthymic temperament $(\beta=-0.21, p<0.01)$ were found to be significant predictors of information stress. Table 3 summarizes the results. The standardized $\beta$ coefficient indicates that the higher the level of cyclothymic and anxious temperament, the higher the information stress, while the higher the hyperthymic temperament, the lower the information stress. However, cyclothymic and hyperthymic temperaments did not significantly differentiate between men and women, so they were excluded from the mediation analysis.

The mediation model was used to explore whether sex, directly or indirectly (through the anxious temperament), affected information stress in the whole sample. The result (Figure 1) showed a significant, direct effect of sex on information stress $(\beta=-0.17, p<0.001)$. The anxious temperament was a significant partial mediator of the re-

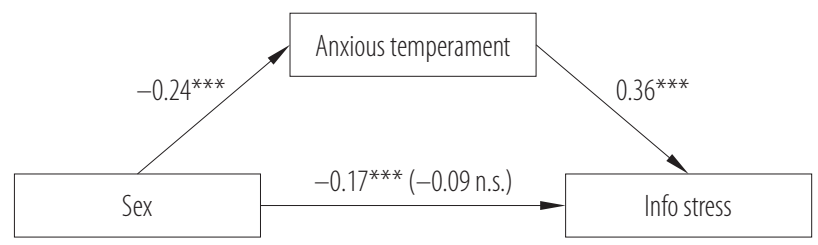

In parentheses, the effect of sex on information stress upon the mediator included into the model.

Figure 1. Relationship between sex and information stress, as mediated by the anxious temperament, in the whole sample in the study conducted in 2017-2018 in Warsaw 
Table 3. Hierarchical multiple regression analysis of sex and affective temperaments as predictors of information stress in the whole sample $(\mathrm{N}=231)$ in the study conducted in 2017-2018 in Warsaw

\begin{tabular}{lcccccc}
\hline \multicolumn{1}{c}{ Variable } & $\mathrm{B}$ & $\mathrm{SEB}$ & $\beta$ & $\mathrm{R}^{2}$ & $\Delta \mathrm{R}^{2}$ & $\begin{array}{c}\text { Semi-partial } \\
\text { correlations }\end{array}$ \\
\hline $\begin{array}{l}\text { Step 1 } \\
\text { Sex }\end{array}$ & -5.63 & 2.15 & $-0.17^{* *}$ & & \\
Step 2 & & & 0.02 & -0.17 \\
sex & -3.34 & 2.11 & -0.10 & & $0.18^{* * *}$ & \\
depressive & -11.82 & 9.24 & -0.12 & & -0.09 \\
cyclothymic & 22.49 & 6.75 & $0.28^{* * *}$ & & -0.08 \\
hyperthymic & -16.15 & 5.68 & $-0.21^{* *}$ & & 0.20 \\
irritable & -8.47 & 6.76 & -0.10 & & -0.17 \\
anxious & 18.77 & 6.67 & $0.25^{* *}$ & & -0.07 \\
\hline
\end{tabular}

${ }^{* *} \mathrm{p}<0.01 ;{ }^{* * *} \mathrm{p}<0.001$

lationship between sex and information stress (Sobel test values: $\mathrm{Z}=-3.14, \mathrm{p}<0.001)$.

The higher the anxious temperament level in women, the higher the information stress in that group, while the lower the anxious temperament level in men, the lower the information stress in that group.

\section{DISCUSSION}

This study aimed to understand the relationships between sex and the level of information stress, as mediated by affective temperaments. The authors hypothesized that sex might affect the level of information stress directly and indirectly, through the mediation of affective temperaments. The authors further expected that anxious temperament would be a significant mediator between sex and the level of information stress.

The results showed that sex might directly affect information stress, but that effect tended to be weak. At the same time, women showed significantly higher anxious temperament levels, and significantly higher information stress, compared to men, suggesting that they were more involved in negative emotional reactions to information overload. Information stress turned out to be stronger, to a statistically significant degree, in the experience of women than men. This result was already obtained in earlier studies on information stress [30], and it may illustrate a more general relationship between sex and anxiety under psychological stress. Using transactional theory of stress and coping [35], information overload may serve as one of the greatest stressors that can cause information anxiety resulting from the inability to process information [8]. Marques et al. [36] conducted a review based on animal models, suggesting that women were more susceptible to stress, compared to men, due to neurophysiological mechanisms functioning (e.g., adult hippocampal neurogenesis), as well as gonadal hormones and their fluctuations. In turn, in their studies in mice, Pfau et al. [37] paid attention to the importance of genetic factors in womenmen stress reactions. According to the authors, women engage different molecular processes in brain structures in response to stress than men do, which leads to differences between the sexes in susceptibility to stress.

Biological factors are not the only ones that explain the differences between women and men in terms of stress reactivity. Dedovic et al. [38] hypothesized an important role of gender socialization in human adults' stress reac- 
tivity. According to the authors, early gender socialization and social learning are the key to understand differences in stress responsivity between men and women. A more restrictive way of raising girls, compared to boys, may weaken their sense of control and increase their sense of dependence on others. Mazure and Maciejewski [39] suggested that women might rate higher on 2 cognitive styles defined as "concern about disapproval" and "need for control" than men, so they might be at a greater risk of affective disorders than men due to these cognitive styles, both in the absence and in the presence of stressful life events. In general, women experienced significantly greater emotional reactivity than men [40] and achieved higher scores on the affective temperaments scales, with the exception of hyperthymic (more "adaptive") temperament which was higher in men than women [17,19].

The identification of the negative emotions dominating in other than hyperthymic, i.e., depressive, cyclothymic, irritable and anxious affective temperaments, may help explain these results. The constant inflow of information requires constant selection and ongoing data processing. The work of the mind involves working memory, i.e., attention and short-term memory. The basic psychological mechanism explaining behavior in the information-rich environment remains attention control [41]. Its exercise by an individual is one of the so-called executive functions. This is facilitated by the presence of 3 inhibitory functions that remove unnecessary information from the analysis field in a given situation, blocking the flow of data incompatible with the task being performed and suppressing activities that are not adapted to the problem being solved. Negative emotions, especially anxiety, weaken inhibitory functions, leading to a gradual increase in mental overload by the accumulation of data. They also induce thinking about the concern for success (intrusive), and attempts to eliminate them reduce operational memory. Emo- tions with a negative sign are an additional burden on the cognitive system, weakening its functioning in every situation, and more strongly under information stimulation conditions [42].

The hierarchical multiple regression analysis demonstrated that sex explained only about $2 \%$ of the information stress variance (Table 3 ). When 5 affective temperaments were added into the hierarchical multiple regression analysis, the percentage of the variance accounted for increased to $19 \%$, indicating their significant role in the regulation of responses to information stress. Based on the regression coefficients, only 3 variables were found to be significant predictors of information stress assessment: cyclothymic, hyperthymic and anxious temperaments. However, cyclothymic and hyperthymic temperaments do not differentiate between men and women (Table 1), so they were excluded from further analysis. Standardized $\beta$ coefficients indicate that the higher the level of anxious temperament, the higher the level of information stress. The mediation analysis (Figure 1) showed that anxious temperament might serve as a significant mediator between sex and the level of information stress.

Interpretation of these results must take into account several potential limitations of the study. This research was cross-sectional in nature, which limits the possibility of drawing conclusions about the causal links between sex, affective temperaments and information stress. Sex or gender differences may play a significant role in information stress, but further longitudinal studies are needed to identify its impact. The estimates of the variables were made using self-report measures, which are highly susceptible to a number of biases. No cognitive abilities or the occurrence of affective disorders were controlled.

Despite these limitations, the study highlighted the relationship between sex and information stress, including the role of anxious affective temperament as a mediator. 
The obtained results have - apart from the cognitive valuethe application value. The ability to manage information is an important psychological competence. Its significance is confirmed by the presence of scientific specialties referred to as information architecture and specialists called information brokers. Operational efficiency includes selecting information, structuring it, combining it with existing knowledge, and using it to solve current tasks. Processing - as the authors have emphasized - requires energy resources that psychologists associate with temperament. Of the 5 so-called affective temperaments, 4 have been associated with information stress, and 2 reported by the result of regression analysis - cyclothymic and anxious are significant predictors of information stress, increasing its intensity. Anxious as well as cyclothymic temperaments seem particularly unfavorable in the case of work and positions related to the exposure to information stress (politics, journalism, team management). The study findings stress the importance of early screening to identify, as soon as possible, those with high scores in affective temperament and develop interventions to prevent the intensification of information stress, especially in women.

Therefore, it is possible to postulate diagnosis of the affective temperaments in the process of recruiting employees to work in occupations exposed to constant information stimulation. It is also advisable to monitor the stress level of employees and undertake actions aimed at limiting the sources of stress. The task facilitates the availability of techniques and the ease of their application, both at the advisory and selection stage.

Future research should focus on the relationship of affective temperaments with different types of stress in women and men, controlling the affective states of the study participants and its other personality traits associated with stress. Psychophysiological and neuroimaging studies of the relationship between affective temperaments and information overload in women and men would be of particular importance.

\section{CONCLUSIONS}

The female group was characterized with a significantly higher age, information stress, and anxious temperament values, and with a significantly lower irritable temperament value, compared to men. Cyclothymic temperament, anxious temperament and hyperthymic temperament were found to be significant predictors of information stress. The mediation analysis showed a significant direct effect of sex on information stress. The anxious temperament was a significant mediator of the relationship between sex and information stress.

\section{REFERENCES}

1. Eppler MJ, Mengis J. The concept of information overload: A review of literature from organization science, accounting, marketing, MIS, and related disciplines. Inform Soc. 2004; 20(5):325-44, https://doi.org/10.1080/01972240490507974.

2. Levine $\mathrm{N}$. The nature of the glut: Information overload in postwar America. Hist Hum Sci. 2017;30(1):32-49, https:// doi.org/10.1177/0952695116686016.

3. Zajenkowski M, Carelli MG, Ledzińska M. Cognitive processes in time perspective. In: Stolarski M, Fieulaine N, van Beek W, editors. Time perspective theory; Review, research and application: Essays in honor of Philip G. Zimbardo. Cham: Springer; 2015. p. 243-55.

4. Ragu-Nathan TS, Tarafdar M, Ragu-Nathan BS, Tu Q. The consequences of technostress for end users in organizations: Conceptual development and empirical validation. Inf Syst Res. 2008;19(4):417-33, https://doi.org/10.1287/isre.1070.0165.

5. Allen D, Wilson T. Information overload: Context and causes. New Rev Inform Behav Res. 2003;4(1):31-44, https://doi.org/ 10.1080/14716310310001631426.

6. Edmunds A, Morris A. The problem of information overload in business organisations: a review of the literature. Int J Inf Manage. 2000;20(1):17-28, https://doi.org/10.1016/S0268-4012 (99)00051-1.

7. Li T, Li M. An investigation and analysis of information overload in manager's work. iBusiness. 2011;3(1):49-52, https:// doi.org/10.4236/ib.2011.31008. 
8. Bawden D, Robinson L. The dark side of information: overload, anxiety and other paradoxes and pathologies. J Inform Sci. 2009;35(2):180-91, https://doi.org/10.1177/01 65551508095781.

9. Girard J, Allison M. Information anxiety: Fact, fable or fallacy. Electron. J Knowl Manage. 2008;6(2):111-24.

10. Haase RF, Jome LM, Ferreira JA, Santos EJR, Connacher CC, Sendrowitz K. Individual differences in capacity for tolerating information overload are related to differences in culture and temperament. J Cross Cult Psychol. 2014;45(5): 728-51, https://doi.org/10.1177/0022022113519852.

11. Ledzińska M, Postek S. From metaphorical information overflow and overload to real stress: Theoretical background, empirical findings, and applications. Eur Manag J. 2017;35(6):785-93, https://doi.org/10.1016/j.emj.2017.07.002.

12. Akiskal KK, Akiskal HS. The theoretical underpinnings of affective temperaments: Implications for evolutionary foundations of bipolar disorder and human nature. J Affect Disord. 2005;85(1-2):23139, https://doi.org/10.1016/j.jad. 2004.08.002.

13. DeGeorge DP, Walsh MA, Barrantes-Vidal N, Kwapil TR. A three-year longitudinal study of affective temperaments and risk for psychopathology. J Affect Disord 2014;164:94100, https://doi.org/10.1016/j.jad.2014.04.006.

14. Kawamura Y, Akiyama T, Shimada T, Minato T, Umekage T, Noda Y, et al. Six-year stability of affective temperaments as measured by TEMPS-A. Psychopathology. 2010;43(4):24047, https://doi.org/10.1159/000313522.

15. Rovai L, Maremmani AG, Rugani F, Bacciardi S, Pacini M, Dell'Osso L, et al. Do Akiskal \& Mallya's affective temperaments belong to the domain of pathology or to that of normality? Eur Rev Med Pharmacol Sci. 2013;17(15):2065-79.

16. Vázquez GH, Tondo L, Mazzarini L, Gonda X. Affective temperaments in general population: A review and combined analysis from national studies. J Affect Disord. 2012;139(1):18-22, https://doi.org/10.1016/j.jad.2011.06.032.

17. Blöink R, Brieger P, Akiskal HS, Marneros A. Factorial structure and internal consistency of the German TEMPS-A scale: Validation against the NEO-FFI questionnaire. J Affect Disord. 2005;85(1-2):77-83, https://doi.org/10.1016/ S0165-0327(03)00101-0.

18. Kwapil TR, DeGeorge D, Walsh MA, Burgin CJ, Silvia PJ, Barrantes-Vidal N. Affective temperaments: Unique constructs or dimensions of normal personality by another name? J Affect Disord. 2013;151(3):882-90, https://doi. org/10.1016/j.jad.2013.07.028.

19. Oniszczenko W, Stanisławiak E, Dembińska-Krajewska D, Rybakowski J. Regulative Theory of Temperament versus affective temperaments measured by the Temperament Evaluation of Memphis, Pisa, Paris and San Diego AutoQuestionnaire (TEMPS-A): A study in a non-clinical Polish sample. Curr Issues Personal Psychol. 2017;5(2):73-82, https://doi.org/10.5114/cipp.2017.65847.

20. Strelau J. Temperament as a regulator of behavior. After fifty years of research. New York, NY: Werner; 2008.

21. Jeronimus BF, Kotov R, Riese H, Ormel J. Neuroticism's prospective association with mental disorders halves after adjustment for baseline symptoms and psychiatric history, but the adjusted association hardly decays with time: A meta-analysis on 59 longitudinal/prospective studies with 443313 participants. Psychol Med. 2016;46(14):2883-906, https://doi.org/10.1017/S0033291716001653.

22. Rihmer Z, Akiskal KK, Rihmer A, Akiskal HS. Current research on affective temperaments. Curr Opin Psychiatr. 2010;23(1):12-8, https://doi.org/10.1097/YCO.0b013e32833 $299 \mathrm{~d} 4$

23. Lungu $\mathrm{O}$, Potvin S, Tikàsz A, Mendrek A. Sex differences in effective fronto-limbic connectivity during negative emotion processing. Psychoneuroendocrinology. 2015;62:180-88, https://doi.org/10.1016/j.psyneuen.2015.08.012.

24. Allan NP, Judah MR, Albanese BJ, Macatee RJ, Sutton CA, Bachman MD, et al. Gender differences in the relation between the late positive potential in response to anxiety sensitivity images and self-reported anxiety sensitivity. Emotion. 2019;19(1):870-83, https://doi.org/10.1037/ emo0000420. 
25. Bottesi G, Martignon A, Cerea S, Ghisi M. Worry and associated cognitive features in Italian university students: Does gender make a difference? Pers Individ Differ. 2018;126:3843, https://doi.org/10.1016/j.paid.2018.01.016.

26. Bale TL, Epperson CN. Sex differences and stress across the lifespan. Nat Neurosci. 2015;18(10):1413-20, https://doi. org/10.1038/nn.4112.

27. Akiskal HS. Toward a definition of generalized anxiety disorder as an anxious temperament type. Acta Psychiatr Scand. 1998;98(Suppl393):66-73, https://doi.org/10.1111/j.1600-0447. 1998.tb05969.x.

28. Akiskal HS, Akiskal KK, Haykal RF, Manning JS, Connor PD. TEMPS-A: Progress towards validation of a selfrated clinical version of the Temperament Evaluation of the Memphis, Pisa, Paris, and San Diego Autoquestionnaire. J Affect Disord. 2005;85(1-2):3-16, https://doi.org/10.1016/ j.jad.2004.12.001.

29. Borkowska A, Rybakowski JK, Drożdż W, Bieliński M, Kosmowska M, Rajewska-Rager A, et al. Polish validation of the TEMPS-A: The profile of affective temperaments in a college student population. J Affect Disord. 2010;123(1-3):3641, https://doi.org/10.1016/j.jad.2009.09.024.

30. Ledzińska M. Człowiek współczesny w obliczu stresu informacyjnego [Modern man in the face of information stress]. Warszawa: Wydawnictwo Instytutu Psychologii PAN; 2009. Polish.

31. IBM Corp. IBM SPSS Statistics for Windows, Version 24.0. Armonk, NY: IBM Corp.; 2015.

32. Tabachnick BG, Fidell LS. Using Multivariate Statistics. 6th ed. Boston, MA: Pearson; 2013.

33. Baron R, Kenny DA. The moderator-mediator variable distinction in social psychological research: Conceptual, strategic, and statistical considerations. J Pers Soc Psychol.
1986;51(6):1173-82, https://doi.org/10.1037/0022-3514.51.6. 1173.

34. Cohen J. Statistical power analysis for the behavioral sciences. 2nd ed. Hillsdale, NJ: Erlbaum; 1988.

35. Lazarus RS, Folkman S. Stress, appraisal, and coping. New York, NY: Springer; 1984.

36. Marques AA, Bevilaqua MCdN, da Fonseca, AMP, Nardi AE, Thuret S, Dias GP. Gender differences in the neurobiology of anxiety: Focus on adult hippocampal neurogenesis. Neural Plast. 2016;ID 5026713, https://doi.org/10. 1155/2016/5026713.

37. Pfau ML, Purushothaman I, Feng J, Golden SA, Aleyasin H, Lorsch ZS, et al. Integrative analysis of sex-specific microRNA networks following stress in Mouse Nucleus Accumbens. Front Molec Neurosci. 2016;9:144, https://doi.org/10.3389/ fnmol.2016.00144.

38. Dedovic K, Wadiwalla M, Engert V, Pruessner JC. The role of sex and gender socialization in stress reactivity. Dev Psychol. 2009;45(1):45-55, https://doi.org/10.1037/a0014433.

39. Mazure CM, Maciejewski PK. The interplay of stress, gender and cognitive style in depressive onset. Arch Womens Ment Health. 2003;6(1):5-8, https://doi.org/10.1007/s00737002-0161-3.

40. Husky MM, Mazure CM, Maciejewski PK, Swendsen JD. Past depression and gender interact to influence emotional reactivity to daily life stress. Cogn Ther Res. 2009;33(3):26471, https://doi.org/10.1007/s10608-008-9212-z.

41. Papageorgiu C, Wells A, editors. Rumination: Nature, theory and treatment. Chichester, UK: Wiley; 2004.

42. Hopko DR, Hunt MK, Armento MEA. Attentional task aptitude and performance anxiety. Int J Stress Manage. 2005;12(4):389-408, https://doi.org/10.1037/1072-5245. 12.4.389.

This work is available in Open Access model and licensed under a Creative Commons Attribution-NonCommercial 3.0 Poland License - http://creativecommons.org/ licenses/by-nc/3.0/pl/deed.en. 